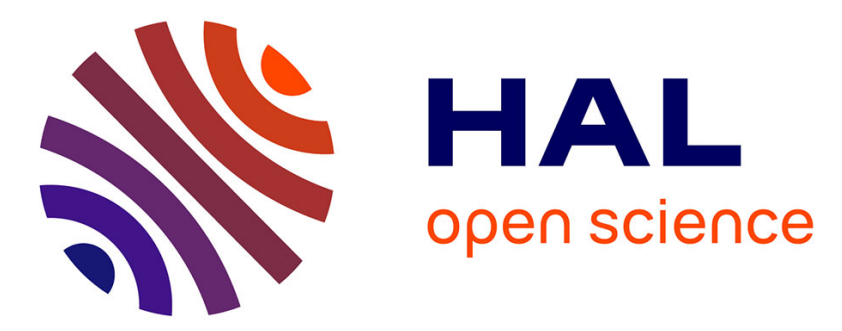

\title{
Evaluating the Uncertainty of a Boolean Formula with Belief Functions
}

\author{
Christelle Jacob, Didier Dubois, Janette Cardoso
}

\section{To cite this version:}

Christelle Jacob, Didier Dubois, Janette Cardoso. Evaluating the Uncertainty of a Boolean Formula with Belief Functions. 14th International Conference on Information Processing and Management of Uncertainty in Knowledge-based Systems (IPMU 2012), Jul 2012, Catane, Italy. pp.521-531, 10.1007/978-3-642-31718-7_54 . hal-03413952

\section{HAL Id: hal-03413952 \\ https://hal.science/hal-03413952}

Submitted on 4 Nov 2021

HAL is a multi-disciplinary open access archive for the deposit and dissemination of scientific research documents, whether they are published or not. The documents may come from teaching and research institutions in France or abroad, or from public or private research centers.
L'archive ouverte pluridisciplinaire HAL, est destinée au dépôt et à la diffusion de documents scientifiques de niveau recherche, publiés ou non, émanant des établissements d'enseignement et de recherche français ou étrangers, des laboratoires publics ou privés. 


\title{
Evaluating the Uncertainty of a Boolean Formula with Belief Functions
}

\author{
Christelle Jacob ${ }^{1,2, \star}$, Didier Dubois ${ }^{2}$, and Janette Cardoso ${ }^{1}$ \\ 1 Institut Supérieur de l'Aéronautique et de l'Espace (ISAE), DMIA department, \\ Campus Supaéro, 10 avenue Édouard Belin - Toulouse \\ 2 Institut de Recherche en Informatique de Toulouse (IRIT), ADRIA department, \\ 118 Route de Narbonne 31062 Toulouse Cedex 9, France \\ \{jacob@isae.fr, dubois@irit.fr, cardoso@isae.fr\}
}

\begin{abstract}
In fault-tree analysis, probabilities of failure of components are often assumed to be precise and the events are assumed to be independent, but this is not always verified in practice. By giving up some of these assumptions, results can still be computed, even though it may require more expensive algorithms, or provide more imprecise results. Once compared to those obtained with the simplified model, the impact of these assumptions can be evaluated. This paper investigates the case when probability intervals of atomic propositions come from independent sources of information. In this case, the problem is solved by means of belief functions. We provide the general framework, discuss computation methods, and compare this setting with other approaches to evaluating the uncertainty of formulas.
\end{abstract}

Keywords: Fault-trees, Belief functions, Boolean satisfaction.

\section{Introduction}

One of the objectives of safety analysis is to evaluate the probabilities of dreadful events. In an analytical approach, this dreadful event is described as a Boolean function $F$ of some atomic events, that represent the failures of the components of a system, or possibly some of its configuration states. This method requires that all probabilities of elementary component failures or configuration states be known and independent, in order to compute the probability of the dreadful event. But in real life scenarios, those assumptions are not always verified. This study takes place in the context of maintenance and dependability studies (Airbus project @MOST) in aviation business.

In this paper, we first investigate different approaches using interval computations in order to compute the probability of a Boolean expression in terms of the probabilities of its literals, a problem of direct relevance in fault-tree analysis. The usual assumptions that probabilities of literals are known and the corresponding events are independent are removed. We consider the situation when

^ C. Jacob has a grant supported by the @MOST Prototype, a joint project of Airbus, IRIT, LAAS, ONERA and ISAE. 
knowledge about probabilities is incomplete (only probability intervals are available), and envisage two assumptions about independence: first the case when no assumption is made about dependence between events represented by atoms, and then the case when the probability intervals come from independent sources of information. We more specifically investigate the use of belief functions to model the latter case, taking advantage of the fact that imprecise probabilities on a binary set are belief functions. We give results on the form of the global belief function resulting from applying Dempster rule of combination to atomic belief functions. We provide results on the computation of belief and plausibility of various kinds of propositional formulas, as found in the application to fault tree analysis. We compare the obtained results with those obtained in other scenarios (stochastic independence between atoms, and the no independence assumption).

\section{Evaluation of the Probability of a Boolean Expression}

Let $\mathcal{X}$ be a set of Boolean variables $x_{1}, \ldots, x_{n}$ such that $x_{i} \in \Omega_{i}=\left\{A_{i}, \neg A_{i}\right\}$; $A_{1}, \ldots, A_{n}$ denote atomic symbols associated to elementary faults or configuration states of a system. We denote by $\Omega=\prod_{i=1}^{n}\left\{A_{i}, \neg A_{i}\right\}$ the set of interpretations $\mathcal{X} \rightarrow\{0,1\}$. An element $\omega \in \Omega$ is also called minterm, and it corresponds to a stochastic elementary event. It can also be interpreted as describing the state of the world at a given time. It can be written both as a maximal conjunction of literals or denoted by the set of its positive literals (it is Herbrand's notation). Let $F$ be a Boolean formula expressed by means of the variables $x_{i}$ : its models form a subset $[F]$ of $\Omega$, the set of states of the world where $F$ is true; also called the set of minterms of $F$. Hence, the probability of $F, P(F)$, can be written as the sum:

$$
P(F)=\sum_{\omega \in[F]} p(\omega)
$$

where $p(\omega)$ stands for $P(\{\omega\})$. When the independence of the $x_{i}$ 's is assumed (i.e. $A_{i}$ independent of $A_{j}, \forall i \neq j$ ), this sum becomes:

$$
P(F)=\sum_{\omega \in[F]}\left[\prod_{A_{i} \in \mathcal{L}_{\omega}^{+}} P\left(A_{i}\right) \prod_{A_{i} \in \mathcal{L}_{\omega}^{-}}\left(1-P\left(A_{i}\right)\right)\right]
$$

where $\mathcal{L}_{\omega}^{+}$is the set of positive literals of $\omega$ and $\mathcal{L}_{\omega}^{-}$the set of its negative literals.

In the case where $P\left(A_{i}\right)$ is only known to lie in an interval, i.e. $P\left(A_{i}\right) \in$ $\left[l_{i}, u_{i}\right], i=1 \ldots n$, the problem is to compute the tightest range $\left[l_{F}, u_{F}\right]$ containing the probability $P(F)$. Let $\mathscr{P}$ be the convex probability family $\left\{P, \forall i P\left(A_{i}\right) \in\right.$ $\left.\left[l_{i}, u_{i}\right]\right\}$ on $\Omega$. In the following, we shall formally express this problem under various assumptions concerning independence.

\subsection{Without Any Independence Hypothesis}

Without knowledge about the dependency between the $x_{i}, i=1 \ldots n$, finding the tightest interval for the range $\left[l_{F}, u_{F}\right]$ of $P(F)$ boils down to a linear optimization 
problem under constraints. This goal is achieved by solving the two following problems:

$$
l_{F}=\min \left(\sum_{\omega \models F} p(\omega)\right) \text { and } u_{F}=\max \left(\sum_{\omega \models F} p(\omega)\right)
$$

under the constraints $l_{i} \leq \sum_{\omega \models A_{i}} p(\omega) \leq u_{i}, i=1 \ldots n$ and $\sum p(\omega)=1$.

Solving each of those problems can be done by linear programming with $2^{n}$ unknown variables $p(\omega)$. It is a particular case of the probabilistic satisfiability problem studied in [3], where known probabilities are attached to sentences instead of just atoms.

\subsection{When Variables $x_{i}$ Are Stochastically Independent}

In the case where the independence of the $x_{i}, i=1 \ldots n$, is assumed,

$$
p(\omega)=\prod_{i=1}^{n} P\left(x_{i}(\omega)\right)
$$

where: $x_{i}(\omega)=\left\{\begin{array}{l}A_{i} \text { if } \omega \models A_{i} \\ \neg A_{i} \text { otherwise }\end{array}\right.$. The corresponding probability family $\mathscr{P}_{I}=$ $\left\{\prod_{i=1}^{n} P_{i} \mid P_{i}\left(\left\{A_{i}\right\}\right) \in\left[l_{i}, u_{i}\right]\right\}$, where $P_{i}$ is a probability measure on $\Omega_{i}$, is not convex. Indeed, take two probability measures $P, P^{\prime} \in \mathscr{P}_{I}, P=\prod_{i=1}^{n} P_{i}$ and $P^{\prime}=\prod_{i=1}^{n} P_{i}^{\prime}$. For $\lambda \in[0,1]$, the sum $\lambda \prod_{i=1}^{n} P_{i}+(1-\lambda) \prod_{i=1}^{n} P_{i}^{\prime} \neq \prod_{i=1}^{n}\left(\lambda P_{i}+(1-\lambda) P_{i}^{\prime}\right)$, so it is not an element of $\mathscr{P}_{I}$.

This assumption introduces some non-linear constraints in the previous formulation, hence the previous methods (section 2.1) cannot be applied. Instead of a linear problem with $2^{n}$ variables, we now have a non-linear optimization problem with $n$ variables. Interval Analysis can be used to solve it [1].

\section{The Case of Independent Sources of Information}

When there is no knowledge about the dependency between the $x_{i}$ 's, but the information about $P\left(A_{i}\right)$ comes from independent sources, belief functions can be used to solve the problem of probability evaluation. The information $P\left(A_{i}\right) \in$ $\left[l_{i}, u_{i}\right]$ is totally linked to its source. $l_{i}$ can be seen as the degree of belief of $A_{i}$ and $u_{i}$ as its plausibility: $l_{i}=\operatorname{Bel}\left(A_{i}\right)$ and $u_{i}=\operatorname{Pl}\left(A_{i}\right)$ in the sense of Shafer.

Proposition 1. The interval $\left[l_{i}, u_{i}\right]$ defines a unique belief function on $\Omega_{i}$. 
Proof: To see it we must find a unique mass assignment and the solution is:

- $\operatorname{Bel}\left(\left\{A_{i}\right\}\right)=l_{i}=m^{i}\left(\left\{A_{i}\right\}\right)$;

- $\operatorname{Pl}\left(\left\{A_{i}\right\}\right)=1-\operatorname{Bel}\left(\left\{\neg A_{i}\right\}\right)=u_{i} \Longrightarrow m^{i}\left(\left\{\neg A_{i}\right\}\right)=\operatorname{Bel}\left(\left\{\neg A_{i}\right\}\right)=1-u_{i}$;

- The sum of masses is $m^{i}\left(\left\{A_{i}\right\}\right)+m^{i}\left(\left\{\neg A_{i}\right\}\right)+m^{i}\left(\Omega_{i}\right)=1$, so $m^{i}\left(\Omega_{i}\right)=u_{i}-l_{i}$.

We call such $m^{i}$ atomic mass functions. In order to combine two independent mass functions, Dempster rule of combination should be used.

\section{Definition 1 (Dempster-Shafer rule)}

For two masses $\mathrm{m}^{1}$ and $\mathrm{m}^{2}$, the joint mass $\mathrm{m}^{1,2}$ can be computed as follows:

- $m^{1,2}(\varnothing)=0$

- $m^{1,2}(S)=\frac{\sum_{B \cap C=S} m^{1}(B) m^{2}(C)}{1-\sum_{B \cap C=\varnothing} m^{1}(B) m^{2}(C)}, \forall S \subseteq \Omega$

In our problem, each source gives an atomic mass function, and there are $n$ sources, so the mass function over all $\Omega$ is : $m_{\Omega}=m^{1} \oplus \cdots \oplus m^{n}$. To find this $m_{\Omega}$ for $n$ atomic mass functions, we can use the associativity of Dempster rule of combination. Here, $A_{i}, i=1, \ldots, n$ are atomic symbols, they are always compatible, i.e. $A_{i} \wedge A_{j} \neq \varnothing$ for all $A_{i}, A_{j}, i \neq j$. So the denominator is one in the above equation.

A focal element of $m^{\Omega}$ is made of a conjunction of terms of the form $A_{i}, \neg A_{j}$ and $\Omega_{k}$ (which is the tautology), for $i \neq j \neq k$. Hence it is a partial model. Let $\mathcal{P}(F)$ bet the set of partial models $\phi$ of a Boolean formula $F$, that are under the form of conjunction of elements $\lambda_{i} \in\left\{A_{i}, \neg A_{i}, \Omega_{i}\right\}: \phi={ }_{i=1, \ldots, n}^{\wedge} \lambda_{i}$. Then, $\mathcal{P}(F)=\left\{\phi=\wedge_{A_{i} \in \mathcal{L}_{\phi}^{+}} A_{i} \wedge_{\neg A_{i} \in \mathcal{L}_{\phi}^{-}} \neg A_{i}=F\right\}$, with $\mathcal{L}_{\phi}^{+}$(resp. $\mathcal{L}_{\phi}^{-}$) the set of positive (resp. negative) literals of $\phi$.

\section{Proposition 2 (Combination of $\mathbf{n}$ atomic mass functions)}

For $n$ atomic masses $m^{i}, i=1, \ldots, n$ on $\Omega_{i}$, the joint mass $m^{\Omega}$ on $\Omega$ can be computed as follows for any partial model $\phi$ :

$$
m^{\Omega}(\phi)=\prod_{i \in \mathcal{L}_{\phi}^{+}} l_{i} \prod_{i \in \mathcal{L}_{\phi}^{-}}\left(1-u_{i}\right) \prod_{i \notin \mathcal{L}_{\phi}}\left(u_{i}-l_{i}\right)
$$

This modeling framework differs from the usual one when atomic variables are supposed to be stochastically independent. Here, the independence assumption pertains to the sources of information, not the physical variables.

\section{The Belief and Plausibility of a Boolean Formula}

The belief of a Boolean formula $F$, of the form $\operatorname{Bel}(F)=\sum_{\phi \models F} m_{\Omega}(\phi)$, theoretically requires $3^{n}$ computations due to the necessity of enumerating the partial 
models for $n$ atomic variables. Indeed, all conjunctions $\phi={ }_{i=1, \ldots, n}^{\wedge} \lambda_{i}$ must be checked for each $\lambda_{i} \in\left\{A_{i}, \neg A_{i}, \Omega_{i}\right\}$. Verifying that a partial model implies $F$ also requires $2^{n}$ computations. Plausibility computation, given by the equation $P l(F)=\sum_{S \wedge \phi \neq \varnothing} m_{\Omega}(\phi)$ requires to determine partial models not incompatible with $F$. From the partial models, it will need at most $2^{n}$ computation. But it can also be computed by using the duality of belief and plausibility given by:

$$
\operatorname{Pl}(F)=1-\operatorname{Bel}(\neg F)
$$

Example 1. Belief functions of the disjunction $F=A_{1} \vee A_{2}$

\begin{tabular}{|c|c|c|c|}
\hline & $A_{1}$ & $\neg A_{1}$ & $\Omega_{1}$ \\
\hline$A_{2}$ & $A_{1} \wedge A_{2}$ & $\neg A_{1} \wedge A_{2}$ & $A_{2}$ \\
& $l_{1} l_{2}$ & $\left(1-u_{1}\right) l_{2}$ & $\left(u_{1}-l_{1}\right) l_{2}$ \\
\hline$\neg A_{2}$ & $A_{1} \wedge \neg A_{2}$ & $\neg A_{1} \wedge \neg A_{2}$ & $\neg A_{2}$ \\
& $l_{1}\left(1-u_{2}\right)$ & $\left(1-u_{1}\right)\left(1-u_{2}\right)$ & $\left(u_{1}-l_{1}\right)\left(1-u_{2}\right)$ \\
\hline$\Omega_{2}$ & $A_{1}$ & $\neg A_{1}$ & $\Omega$ \\
& $l_{1}\left(u_{2}-l_{2}\right)$ & $\left(1-u_{1}\right)\left(u_{2}-l_{2}\right)$ & $\left(u_{1}-l_{1}\right)\left(u_{2}-l_{2}\right)$ \\
\hline
\end{tabular}

Partial models that imply $F$ are $\left\{A_{1}, A_{2}, A_{1} \wedge \neg A_{2}, A_{2} \wedge \neg A_{1}, A_{1} \wedge A_{2}\right\}$, so: $\operatorname{Bel}(F)=\left(u_{1}-l_{1}\right) l_{2}+l_{1}\left(u_{2}-l_{2}\right)+l_{1} l_{2}+l_{1}\left(1-u_{2}\right)+l_{2}\left(1-u_{1}\right)=l_{1}+l_{2}-l_{1} l_{2}$, that also reads $1-\left(1-l_{1}\right)\left(1-l_{2}\right)$. Likewise, partial models that are compatible with $F$ are $\left\{A_{1} \wedge A_{2}, \Omega, A_{1}, A_{2}, \neg A_{1}, \neg A_{2}, A_{1} \wedge \neg A_{2}, A_{2} \wedge \neg A_{1}\right\}$, hence $\operatorname{Pl}(F)=$ $u_{1}+u_{2}-u_{1} u_{2}=1-\left(1-u_{1}\right)\left(1-u_{2}\right)$.

\subsection{Conjunctions and Disjunctions of Literals}

In the more general case, we can compute the belief and plausibility of conjunctions and disjunctions of literals indexed by $K \subseteq\{1, \ldots, n\}$.

Proposition 3. The belief of a conjunction $C$, and that of a disjunction $D$ of literals $x_{i}, i \in K$ are respectively given by:

$$
\operatorname{Bel}(C)=\prod_{i \in \mathcal{L}_{\mathcal{C}}^{+}} l_{i} \prod_{i \in \mathcal{L}_{\mathcal{C}}^{-}}\left(1-u_{i}\right) ; \quad \operatorname{Bel}(D)=1-\prod_{i \in \mathcal{L}_{\mathcal{D}}^{+}}\left(1-l_{i}\right) \prod_{i \in \mathcal{L}_{\mathcal{D}}^{-}} u_{i} .
$$

We can deduce the plausibility of conjunctions and disjunctions of literals, noticing that

$$
\operatorname{Bel}\left(\vee_{i \in \mathcal{L}^{+}} A_{i} \vee \vee_{i \in \mathcal{L}^{-}} \neg A_{i}\right)=1-P l\left(\wedge_{i \in \mathcal{L}^{+}} \neg A_{i} \wedge \wedge_{i \in \mathcal{L}^{-}} A_{i}\right)
$$

Proposition 4. The plausibility of a conjunction $C$, and that of a disjunction $D$ of literals $x_{i}, i \in K$ are respectively given by:

$$
P l(C)=\prod_{i \in \mathcal{L}_{\mathcal{C}}^{+}}\left(1-l_{i}\right) \prod_{i \in \mathcal{L}_{\mathcal{C}}^{-}} u_{i} ; \quad P l(D)=1-\prod_{i \in \mathcal{L}_{\mathcal{D}}^{+}} l_{i} \prod_{i \in \mathcal{L}_{\mathcal{D}}^{-}}\left(1-u_{i}\right)
$$




\subsection{Application to Fault-Trees}

Definition 2 (Fault-tree). A fault-tree is a graphical representation of chains of events leading to a dreadful event (failure).

Classical fault-trees are a graphical representation dedicated to Boolean functions that are representable by means of two operators $\vee(\mathrm{OR})$ and $\wedge(\mathrm{AND})$.

Only few applications of Dempster-Shafer theory to fault-Tree Analysis are reported in literature. Limbourg et al. 2] created a Matlab toolbox where each probability is modeled by a random interval on $[0,1]$. Instead of Dempster rule, they use Weighted average [4] for the aggregation of the belief functions of different variables. Murtha 5] uses the same method in an application to small unmanned aerial vehicles. Another method using 3 -valued logic proposed by Guth [6] is compared by Cheng to interval computation, over small examples of Fault-trees [7. The above results can be specialized to fault trees.

A path in a fault tree links the top (dreadful) event to the leaves of the tree: it is called a cut. When this path has a minimal number of steps, it is said to be a minimal cut. Each cut is a conjunction of atoms. As a consequence of the above results we can compute the belief and plausibility of conjunctions and disjunction of $k$ atoms $A_{1}, \ldots A_{k}$ :

$$
\begin{array}{ll}
\operatorname{Bel}(C)=\prod_{i=1}^{k} l_{i}, & \operatorname{Pl}(C)=\prod_{i=1}^{k} u_{i} \\
\operatorname{Bel}(D)=1-\prod_{i=1}^{k}\left(1-l_{i}\right), & P l(D)=1-\prod_{i=1}^{k}\left(1-u_{i}\right) .
\end{array}
$$

From a Fault-tree $F$, an approximation can be obtained by means of minimal cuts. For a given order (maximal number of atoms in conjunctions), appropriate software can find the set of all Minimal Cuts that lead to the top event. The disjunction of all those Minimal Cuts will give us a partial Fault-tree which will be an approximation of F. Fig. 1 is an example of such a Partial Fault-tree.

The Boolean formula $F^{\prime}$ represented by this tree will always be under the form of a disjunction of conjunctions of atoms $C_{1} \vee \ldots \vee C_{m}$. The formula written in this form will be referred to as a Disjunctive Atomic Normal Form (DANF) (excluding

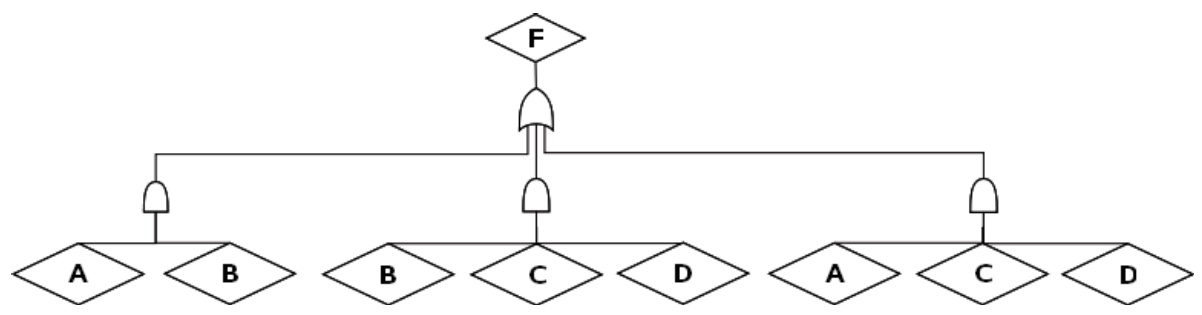

Fig. 1. Example of Partial Fault Tree 
negative literals). In order to compute the Belief function of such a formula, we should generalize the computation of the belief of a disjunction of $k$ atoms.

Proposition 5. [Belief of a disjunctive atomic normal form (DANF)]

$$
\begin{array}{r}
\operatorname{Bel}\left(C_{1} \vee \ldots \vee C_{m}\right)=\sum_{i=1}^{m} \operatorname{Bel}\left(C_{i}\right)-\sum_{i=1}^{m-1} \sum_{j=i+1}^{m} \operatorname{Bel}\left(C_{i} \wedge C_{j}\right) \\
+\sum_{i=1}^{m-2} \sum_{j=i+1}^{m-1} \sum_{k=j+1}^{m} \operatorname{Bel}\left(C_{i} \wedge C_{j} \wedge C_{k}\right)-\ldots+(-1)^{m+1} \operatorname{Bel}\left(C_{1} \wedge \ldots \wedge C_{m}\right),
\end{array}
$$

where $C_{i}$ are conjunctions of atoms.

During the computation, the conjunctions of conjunctions, such as $C_{i} \wedge C_{j} \wedge C_{k}$ must be simplified, deleting redundant atoms. Note that this apparent additivity of a generally non-additive function is due to the specific shape of focal elements (partial models). In general, for $S$ and $T$ Boolean formulas, we cannot write $\operatorname{Bel}(S \vee T)=\operatorname{Bel}(S)+\operatorname{Bel}(T)-\operatorname{Bel}(S \wedge T)$, because there are focal elements in $S \vee T$ that are subsets of neither $S$ nor $T$ nor $S \wedge T$. Here due to the DANF form, all partial models of $C_{1} \vee \ldots \vee C_{m}$ are conjunctions of literals appearing in the conjunctions.

A similar result holds for computing the plausibility of a DNF.

\section{Proposition 6 (Pl of a disjunctive atomic normal form (DANF)).}

$$
\begin{array}{r}
P l\left(C_{1} \vee \ldots \vee C_{m}\right)=\sum_{i=1}^{m} \operatorname{Pl}\left(C_{i}\right)-\sum_{i=1}^{m-1} \sum_{j=i+1}^{m} P l\left(C_{i} \wedge C_{j}\right) \\
+\sum_{i=1}^{m-2} \sum_{j=i+1}^{m-1} \sum_{k=j+1}^{m} P l\left(C_{i} \wedge C_{j} \wedge C_{k}\right)-\ldots+(-1)^{m+1} P l\left(C_{1} \wedge \ldots \wedge C_{m}\right),
\end{array}
$$

where $C_{i}$ are conjunctions of literals.

Thanks to the duality between Belief and Plausibility, both computations are quite similar, hence the time complexity does not increase. It is also much less time consuming than an exhaustive computation as presented in section 3 .

\subsection{General Case}

The general case of a Boolean formula with positive and negative literals is more tricky. Such a formula can appear, for example, in fault-trees representing different modes in a system, or representing exclusive failures [1]. Of course we can assume the formula is in DNF format. But it will be a conjunction of literals, and it is no longer possible to apply the two previous propositions. Indeed when conjunctions contain opposite literals, they have disjoint sets of 
models but their disjunctions may be implied by partial models (focal elements) that imply none of the conjuncts. For instance consider $A \vee(\neg A \wedge B$ ) (which is just the disjunction $A \vee B$ we know how to deal with). It does not hold that $\operatorname{Bel}(A \vee(\neg A \wedge B)=\operatorname{Bel}(A)+\operatorname{Bel}(\neg A \wedge B)$, since the latter sum neglects $m(B)$, where $B$ is a focal element that implies neither $A$ nor $\neg A \wedge B$. However if $C_{1} \vee \ldots \vee C_{m}$ are pairwise mutually inconsistent partial models such that no disjunction of $C_{i}$ and $C_{j}$ contains a partial model implying neither $C_{i}$ nor $C_{j}$, the computation can be simplified since then $\operatorname{Bel}\left(C_{1} \vee \ldots \vee C_{m}\right)=\sum_{i=1}^{m} \operatorname{Bel}\left(C_{i}\right)$. For instance, the belief in an exclusive OR $\operatorname{Bel}\left(\left(A_{1} \wedge \neg A_{2}\right) \vee\left(A_{2} \wedge \neg A_{1}\right)\right)$ is of this form. More work is needed in the general case.

\section{Comparison between Interval Analysis and Dempster-Shafer Theory}

Table 1 summarizes the results obtained using the two methods seen in section 2.2 and 3 applied to Boolean formulas: (i) the belief functions method with the assumption that the probability values come from independent sources of information, and (ii) the full-fledged interval analysis method under the assumption that all atomic events are independent 11. These two assumptions do not reflect the same kind of situations. In particular the independence between sources of information may be justified if elementary components in the device under study are different from one another, which often implies that the sources of information about them will be distinct. However the fact that such elementary components interact within a device tends to go against the statistical independence of their respective behaviors.

Those results are given for the basic Boolean operators with variables $\mathrm{A}, \mathrm{B}$, $\mathrm{C}$ and D. The probability interval used for those computations are: $P(A) \in$ $[0.3,0.8], P(B) \in[0.4,0.6], P(C) \in[0.2,0.4]$, and $P(D) \in[0.1,0.5]$.

Table 1. Comparison between Interval Analysis and Dempster-Shafer Theory

\begin{tabular}{|c|c|c|c|}
\hline Connective & Formula & Belief Functions (i) & Interval Analysis (ii) \\
\hline OR & $A \vee B$ & $\begin{array}{l}l_{F}=l_{A}+l_{B}-l_{A} l_{B}=0.58 \\
u_{F}=u_{A}+u_{B}-u_{A} u_{B}=0.92\end{array}$ & $\begin{array}{l}l_{F}=l_{A}+l_{B}-l_{A} l_{B}=0.58 \\
u_{F}=u_{A}+u_{B}-u_{A} u_{B}=0.92\end{array}$ \\
\hline AND & $A \wedge B$ & $\begin{array}{l}l_{F}=l_{A} l_{B}=0.12 \\
u_{F}=u_{A} u_{B}=0.48\end{array}$ & $\begin{array}{l}l_{F}=l_{A} l_{B}=0.12 \\
u_{F}=u_{A} u_{B}=0.48\end{array}$ \\
\hline IMPLIES & $A \Rightarrow B$ & $\begin{array}{l}l_{F}=l_{A}+\left(1-u_{A}\right)\left(1-u_{B}\right)=0.48 \\
u_{F}=1-l_{A}\left(u_{B}-l_{A}\right)=0.94\end{array}$ & $\begin{array}{l}l_{F}=1-u_{A}+l_{B} u_{A}=0.52 \\
u_{F}=1-l_{A}+u_{B} l_{A}=0.88\end{array}$ \\
\hline ExOR & $A \triangle B$ & $\begin{array}{l}l_{F}=l_{A}\left(1-u_{B}\right)+l_{B}\left(1-u_{A}\right) \\
u_{F}=u_{A}+u_{B}-l_{A} l_{B}-u_{A} u_{B} \\
{\left[l_{F}, u_{F}\right]=[0.2,0.8]}\end{array}$ & {$[0.44,0.56]$} \\
\hline $\begin{array}{l}\text { Fault-tree } \\
\text { (Fig. 1) }\end{array}$ & $\mathrm{F}$ & $\begin{array}{l}l_{F}=l_{A} l_{B}+l_{B} l_{C} l_{D}+l_{A} l_{C} l_{D}- \\
2 l_{A} l_{B} l_{C} l_{D} \\
u_{F}=u_{A} u_{B}+u_{B} u_{C} u_{D}+u_{A} u_{C} u_{D}- \\
2 u_{A} u_{B} u_{C} u_{D} \\
{\left[l_{F}, u_{F}\right]=[0.1292,0.568]}\end{array}$ & $\begin{array}{l}l_{F}=l_{A} l_{B}+\left(1-l_{A}\right) l_{B} l_{C} l_{D}+(1- \\
\left.l_{B}\right) l_{A} l_{C} l_{D} \\
l_{F}=u_{A} u_{B}+\left(1-u_{A}\right) u_{B} u_{C} u_{D}+ \\
\left(1-u_{B}\right) u_{A} u_{C} u_{D} \\
{\left[l_{F}, u_{F}\right]=[0.1292,0.568]}\end{array}$ \\
\hline
\end{tabular}


In Interval Analysis, knowing the monotonicity of a formula makes the determination of its range straightforward. A Boolean formula is monotonic with respect to a variable when we can find an expression of the formula where this variable appears only in a positive or negative way. It is the case for the formulas And, Or, and Implies.

But when the monotonicity is not easy to study, an exhaustive computation for all intervals boundaries must be carried out, like for the Equivalence and the Exclusive Or 1 .

The difference between the results varies a lot with the formula under study. Sometimes, using the Dempster-Shafer theory give the same results as interval analysis, hence, in those cases, the dependency assumption does not have a big influence on the output value; e.g in case of conjunction and disjunction of literals, but also disjunction of conjunctions of atoms (as shown in table 11). This is not surprising as focal elements also take the form of conjunctions of literals, and their masses are products of marginals. The fact that in such cases the same results are obtained does not make the belief function analysis redundant: it shows that the results induced by the stochastic independence assumption are valid even when this assumption is relaxed, for some kinds of Boolean formulas. For more general kinds of Boolean formulas, the intervals computed by using belief functions are in contrast wider than when stochastic independence is assumed.

In general, the probability family induced by the stochastic independence assumption will be included in the probability family induced by the belief functions. This proposition can be proved using the results of Fetz [9] and Couso and Moral [10. Any probability measure in $\mathcal{P}(m)=\{P \geq B e l\}$ dominating a belief function induced by a mass function $m$ can be written in the form: $P=\sum_{E \subseteq \Omega} m(E) \cdot P_{E}$ where $P_{E}$ is a probability measure such that $P_{E}(E)=1$ that shares the mass $m(E)$ among elements of $E$. For a function of two Boolean variables $x_{1}$ and $x_{2}$, with two ill-known probability values $P_{1}\left(A_{1}\right)=p_{1}$ and $P_{1}\left(A_{2}\right)=p_{2}, p_{1}$ is of the form $l_{1}+\alpha\left(u_{1}-l_{1}\right)$ for some $\alpha \in[0,1]$ and $p_{2}$ is of the form $l_{2}+\beta\left(u_{2}-l_{2}\right)$ for some $\beta \in[0,1]$. The explicit sharing, among interpretations, of the masses $m(E)$, induced by probability intervals $\left[l_{1}, u_{1}\right]$ and $\left[l_{2}, u_{2}\right]$, that enables $P=P_{1} P_{2}$ to be recovered is:

1. The masses on interpretations bear on singletons, hence do not need to be shared.

2. The masses on partial models are shared as follows

- $m\left(A_{1}\right) \beta$ is assigned to $A_{1} A_{2}, m\left(A_{1}\right)(1-\beta)$ to $A_{1} \neg A_{2}$.

- $m\left(A_{2}\right) \alpha$ is assigned to $A_{1} A_{2}, m\left(A_{2}\right)(1-\alpha)$ to $\neg A_{1} A_{2}$.

- $m\left(\neg A_{1}\right) \beta$ is assigned to $\neg A_{1} A_{2}, m\left(\neg A_{1}\right)(1-\beta)$ to $\neg A_{1} \neg A_{2}$.

- $m\left(\neg A_{2}\right) \alpha$ is assigned to $A_{1} \neg A_{2}, m\left(A_{2}\right)(1-\alpha)$ to $\neg A_{1} \neg A_{2}$.

3. $m(\Omega)$ is shared as follows: $\alpha \beta m(\Omega)$ to $A_{1} A_{2},(1-\alpha) \beta m(\Omega)$ to $\neg A_{1} A_{2}$, $\alpha(1-\beta) m(\top)$ to $A_{1} \neg A_{2},(1-\alpha)(1-\beta) m(\top)$ to $A_{1} A_{2}$. 
It can be checked that the joint probability $P_{1} P_{2}$ is the form $\sum_{E \subseteq \Omega} m(E) \cdot P_{E}$ using this sharing of masses. This result can be extended to more than 2 variables. It indicates that the assumptions of source independence is weaker than the one of stochastic independence, and is of course stronger than no independence assumption at all. So the belief function approach offers a useful and tractable approach to evaluate the impact of stochastic independence assumptions on the knowledge of the probability of dreadful events in fault-tree analysis.

\section{Conclusion}

The more faithful models are to the actual world, the more their complexity increases. When assumptions are made to simplify the model, then it is important to know how far the results stand away from reality in order to use them as appropriately as possible. Having a means to compare different kinds of models and different kinds of assumptions can be a good asset in order to make best decisions out of the models. In this paper, we have laid bare three kinds of assumptions for the calculation of the probability of some risky event in terms of probability of basic atomic formulas. We have focused on the belief function approach that assumes independence between sources of information proving imprecise probabilistic information. We did also gave a formal solution for an application to fault-tree analysis based on a DNF conversion. A practical scalable solution for handling general Boolean formulas is a topic for further research.

\section{References}

1. Jacob, C., Dubois, D., Cardoso, J.: Uncertainty Handling in Quantitative BDDBased Fault-Tree Analysis by Interval Computation. In: Benferhat, S., Grant, J. (eds.) SUM 2011. LNCS, vol. 6929, pp. 205-218. Springer, Heidelberg (2011)

2. Limbourg, P., Savić, R., Petersen, J., Kochs, H.-D.: Fault Tree Analysis in an Early Design Stage using the Dempster-Shafer Theory of Evidence. In: European Safety and Reliability Conference, ESREL 2007, pp. 713-722. Taylor \& Francis Group, Stavanger (2007)

3. Hansen, P., Jaumard, B., Poggi de Aragão, M., Chauny, F., Perron, S.: Probabilistic satisfiability with imprecise probabilities. International Journal of Approximate Reasoning 24(2-3), 171-189 (2000)

4. Ferson, S., Kreinovich, V., Ginzburg, L., Myers, D.S., Sentz, K.: Constructing Probability Boxes and Dempster-Shafer Structures. Sandia Nat. Lab., Albuquerque (2003)

5. Murtha, J.F.: Evidence Theory and Fault-tree Analysis to Cost-effectively improve Reliability in Small UAV Design. Virginia Polytechnic Inst. \& State University

6. Guth, M.A.: A Probability foundation for Vagueness and Imprecision in Fault-tree Analysis. IEEE Trans. Reliability 40(5), 563-570 (1991) 
7. Cheng, Y.-L.: Uncertainties in Fault Tree Analysis. TamKang Journal of Science and Engineering 3(1), 23-29 (2000)

8. Brualdi, R.: Introductory combinatorics, 4th edn., Lavoisier (2004)

9. Fezt, T.: Sets of Joint Probability Measures Generated by Weighted Marginal Focal Sets. In: 2nd Int. Symp. on Imp. Probabilities \& Their Applications, New York (2001)

10. Couso, I., Moral, S.: Independence concepts in evidence theory. International Journal of Approximate Reasoning 51, 748-758 (2010) 